\title{
Le tourteau de coton : effets de la granulation de l'aliment sur les performances de croissance du poulet de chair
}

\author{
Jean-Claude Maliboungou ${ }^{\mathrm{a} *}$, Michel Lessire ${ }^{\mathrm{b}}$, Jean-Marc Hallouis ${ }^{\mathrm{b}}$ \\ a ICRA, Département des systèmes agro-pastoraux, BP 122, Bangui-Lakouanga, \\ République Centrafricaine \\ ${ }^{\mathrm{b}}$ Inra, station de recherches avicoles, 37380 Nouzilly, France
}

(reçu le 9 juillet 1998 ; accepté le 15 décembre 1998)

\begin{abstract}
Effects of feeding mash or pelleted diets based on cottonseed meal on growth performances of broiler chickens. A diet containing $26.7 \%$ cottonseed meal (CSM) was fed to chicks and compared to a control diet based on soyabean meal (SBM). Both diets were offered as meal or pellets. From 7 to 25 days of age, chicks fed diets based on CSM ate less and grew slower $(-13.3$ and $-23.4 \%$, respectively) than those fed diets based on SBM. Chicks fed the CSM-based diet as pellets ate $11.4 \%$ more feed and grew $12.3 \%$ faster than chicks fed the same diet as mash; these parameters were increased by 3 and $7 \%$, respectively, with the SBM-based diet. These results suggest that feed consumption of the CSM-based diet is reduced when fed as mash. However, the CSM-based diet given as pellets remains less efficient than SBM-based pellet diet. (O Elsevier / Inra)
\end{abstract}

cottonseed meal / pelleting / broiler chicken / feed consumption / liveweight gain

Résumé - L'effet de la granulation d'un régime contenant $26,7 \%$ de tourteau de coton a été testé chez le poulet de chair de 7 à $25 \mathrm{j}$ d'âge et comparé à un aliment témoin à base de tourteau de soja. Les poulets nourris avec l'aliment à base de tourteau de coton ont un ingéré alimentaire et un gain de poids vif moyens significativement inférieurs $(-13,3$ et $-23,4 \%$ respectivement $)$ à ceux consommant l'aliment témoin. La granulation de l'aliment coton accroît l'ingéré alimentaire et le poids vif moyen de 11,4 et $12,3 \%$ respectivement, ces paramètres sont seulement augmentés de 3 et $7 \%$ respectivement par la granulation de l'aliment soja. Ces résultats suggèrent que les poulets de chair sous-consomment l'aliment à base de tourteau de coton lorsqu'il est présenté en farine, mais que cet aliment reste moins efficace après granulation comparé à l'aliment soja. (을 Elsevier/Inra)

tourteau de coton / granulation / poulet de chair / ingéré alimentaire / gain de poids vif

\footnotetext{
* Correspondance et tirés à part.
}

Tél. : (00) (236) 616275 


\section{INTRODUCTION}

Les tourteaux de coton constituent une ressource alimentaire potentielle pour l'aviculture tropicale [19] et souvent la seule source protéique disponible. Leur utilisation dans les aliments pour les volailles est très répandue en Afrique [9] et particulièrement en République Centrafricaine. Cependant, des taux d'incorporation supérieurs à $20 \%$ dans les régimes provoquent une réduction des performances de croissance chez le poulet de chair $[1,5,20]$ et de l'intensité de ponte chez la poule pondeuse $[4,17,18]$. Le gossypol, pigment phénolique toxique, le fort taux de glucides pariétaux indigestibles et la faible digestibilité des protéines et de l'énergie des tourteaux de coton sont évoqués comme responsables des effets négatifs de ces tourteaux sur les performances de production des volailles.

En outre, les aliments expérimentaux sont le plus souvent présentés en farine ce qui limite l'ingéré alimentaire et fait augmenter l'indice de consommation. Il est en effet connu depuis longtemps que la granulation peut améliorer de façon substantielle les performances du poulet de chair : $+25 \%$ pour le poids vif, $+15 \%$ pour l'ingéré alimentaire et $-10 \%$ pour l'indice de consommation $[3,8]$, de telles améliorations ne sont cependant plus d'actualité avec les techniques actuelles d'élevage[14] mais restent encore conséquentes. Ces améliorations diminuent lorsque le niveau énergétique de la ration s'accroît [11]. La granulation permet aussi d'inactiver certains facteurs antinutritionnels thermolabiles, certains germes pathogènes et des toxines présents dans les aliments $[10,16]$.

En République Centrafricaine, et dans la plupart des pays d'Afrique tropicale la granulation n'est pas pratiquée. Enfin, dans la plupart des travaux relatifs à l'utilisation du tourteau de coton en alimentation du poulet les aliments sont imparfaitement rééquilibrés en acides aminés indispensables et dans ces conditions on peut attribuer au tourteau de coton les mauvaises performances dues à un déséquilibre protéique.

L'expérience que nous avons réalisée a pour objectif de tester chez le poulet de chair l'effet de la granulation d'un aliment très riche en tourteau de coton sur le gain de poids, l'ingéré et l'efficacité alimentaire. Ces régimes sont équilibrés en acides aminés indispensables bruts et sont comparés à un aliment à base de tourteau de soja ayant les mêmes caractéristiques nutritionnelles brutes.

\section{MATÉRIEL ET MÉTHODES}

Cent vingt poussins de chair mâles de souche SHAVER sont reçus à un jour d'âge. Ils sont pesés individuellement, bagués et séparés en deux lots de poids moyen identique. Pendant la première semaine d'élevage, ils sont élevés en groupe et reçoivent l'un des deux aliments expérimentaux (tableau $I$ ) présenté en farine. Le $7^{\mathfrak{e}}$ jour après une mise à jeun de $7 \mathrm{~h}$, ils sont pesés individuellement, 48 poussins coton et 48 poussins soja sont retenus pour former deux lots homogènes sur la base du poids vif atteint à $7 \mathrm{j}$. Les poussins sont ensuite placés dans des cages individuelles et chaque lot est partagé en deux groupes de 24 animaux de poids identique qui reçoivent alors soit la forme farine soit la forme granulée de l'aliment expérimental distribué la première semaine. Les traitements sont répartis au hasard de chaque côté de la batterie qui comprend quatre étages de 12 cages individuelles.

Les aliments sont distribués du $8^{\mathfrak{e}}$ au $25^{\mathrm{e}}$ jour d'âge. Les compositions et les principales caractéristiques nutritionnelles figurent dans le tableau I. Ces aliments ont les mêmes caractéristiques nutritionnelles brutes calculées, mais diffèrent par la nature de l'apport principal de protéines : tourteau de coton ou tourteau de soja. Les teneurs en acides aminés brutes sont calculées pour être identiques dans les deux aliments. Pour cela, il a été nécessaire de supplémenter l'aliment coton avec de la thréonine et du tryptophane de synthèse. Durant toute la période expérimentale (I à $25 \mathrm{j}$ ), les animaux sont soumis à des conditions standard d'élevage : $24 \mathrm{~h}$ de lumière pendant les deux premiers jours, ensuite $18 \mathrm{~h}$ par jour. Pour les températures, le programme est le suivant : 
$32{ }^{\circ} \mathrm{C}$ les deux premiers jours, $30^{\circ} \mathrm{C}$ du $3^{\mathrm{e}}$ au $7^{\mathrm{e}} \mathrm{j}$, $28^{\circ} \mathrm{C}$ du $8^{\mathrm{e}}$ au $15^{\mathrm{e}} \mathrm{j}$ et $25^{\circ} \mathrm{C}$ du $16^{\mathrm{e}}$ au $25^{\mathrm{e}} \mathrm{j}$.

Les animaux sont pesés individuellement aux âges de 7 et $25 \mathrm{j}$ afin de mesurer le poids vif et le gain de poids. La consommation individuelle des aliments est mesurée aux mêmes âges pour calculer les quantités ingérées et l'indice de consommation. L'ensemble des données recueillies est traité par analyse de variance à deux facteurs (tourteau, présentation) à l'aide du programme Statview. Les analyses des aliments et du tourteau de coton sont effectuées selon les normes Afnor (1986).

\section{RÉSULTATS}

Dès la fin de la première semaine d'élevage, la nature des protéines apportées: tourteau de coton ou tourteau de soja, a un effet significatif $(p<0,0001)$ sur le gain de poids (tableau $I I$ ). Les poussins soja sont plus lourds $(+12 \mathrm{~g})$ que les animaux coton : 153 vs. 141 g. Pendant la période expérimentale en cage ( $8-25 \mathrm{j}$ ), la nature des protéines apportées : tourteau de coton ou tourteau de soja a un effet significatif $(p<0,0001)$

Tableau I. Composition et caractéristiques nutritionnelles des aliments expérimentaux.

\begin{tabular}{|c|c|c|}
\hline & Aliment coton & Aliment soja \\
\hline \multicolumn{3}{|l|}{ Composition (\%) } \\
\hline Maïs & 62,17 & 61,85 \\
\hline Gluten de maïs 60 & 2,08 & - \\
\hline Tourteau de coton ${ }^{1}$ & 26,70 & - \\
\hline Tourteau de soja 48 & - & 31,60 \\
\hline Huile de colza & 4,00 & 2,20 \\
\hline Carbonate de calcium & 1,21 & 1.21 \\
\hline Phosphate Bicalcique & 2,06 & 1,93 \\
\hline Sel & 0,40 & 0,40 \\
\hline Oligo-éléments & 0,10 & 0,10 \\
\hline Vitamines & 0,50 & 0,50 \\
\hline DL Méthionine & 0,13 & 0,14 \\
\hline Lysine $\mathrm{HCl}$ & 0,52 & 0,06 \\
\hline Tryptophane & 0,04 & - \\
\hline Thréonine & 0,09 & - \\
\hline \multicolumn{3}{|l|}{ Caractéristiques nutritionnelles } \\
\hline $\mathrm{EM}\left(\mathrm{kcal} \cdot \mathrm{kg}^{-1}\right)$ & 2950 & 2950 \\
\hline $\operatorname{MAT}(\mathrm{N} \times 6,25)(\%)$ & $20(20)^{2}$ & $20(21,25)$ \\
\hline Lysine (\%) & $1,10(1,13)$ & $1,09(1,17)$ \\
\hline Acides aminés soufrés (\%) & $0,80(0,91)$ & $0,80 \quad(1,01)$ \\
\hline Tryptophane (\%) & 0,23 & 0,23 \\
\hline Thréonine $(\%)$ & $0,76(0,79)$ & $0,76(0,83)$ \\
\hline Calcium $(\%)$ & 0,99 & 0,99 \\
\hline Phosphore disponible (\%) & 0,40 & 0,40 \\
\hline Gossypol libre $(\%)$ & $(0,04)$ & \\
\hline \multicolumn{3}{|l|}{ Densité $\left(\mathrm{kg} \cdot \mathrm{L}^{-1}\right)$} \\
\hline Farine & 0,52 & 0,61 \\
\hline Granulé & 0,65 & 0,70 \\
\hline
\end{tabular}

' Composition du tourteau de coton (\% produit brut) : matière sèche $: 91,80$; protéines brutes : 46,81; matières grasses : 4,38 ; cendres brutes : 6,99 ; gossypol libre : 0,14 ; lysine : 1,83 ; méthionine : 0,73 ; méthionine+cystine : 1,78 ; thréonine : 1,48; leucine : 2,66 ; énergie métabolisable en $\mathrm{kcal} \cdot \mathrm{kg}^{-1}: 1890$ (valeur estimée selon les tables de European table of energy for poultry feedstuffs 1st Edition 1986). 2 Les données entre parenthèses sont mesurées sur les aliments farine. 
Tableau II. Moyenne ( \pm écart type) des poids vif $(\mathrm{g})$, gain de poids $(\mathrm{g})$, ingéré alimentaire ( $\mathrm{g} / \mathrm{animal})$ et l'indice de consommation des poulets selon la composition et la présentation du régime ( $n=24$ poulets par traitement).

\begin{tabular}{|c|c|c|c|}
\hline Période $0-7 \mathbf{j}$ & \multicolumn{3}{|c|}{ Présentation farine } \\
\hline \multicolumn{4}{|l|}{ Poids vif moyen (g) } \\
\hline Aliment coton & \multicolumn{3}{|c|}{$141 \pm 1,5$} \\
\hline Aliment soja & \multicolumn{3}{|c|}{$153 \pm 1,5$} \\
\hline \multirow[t]{2}{*}{ Période 8-25 j } & \multicolumn{2}{|c|}{ Présentation } & \multirow[b]{2}{*}{ Moyenne } \\
\hline & Farine & Granulé & \\
\hline \multicolumn{4}{|c|}{ Poids vif moyen à $25 \mathrm{j}(\mathrm{g})$} \\
\hline Aliment coton & $748 \pm 17$ & $840 \pm 19,5$ & $793 \pm 14^{\mathrm{B}}$ \\
\hline Aliment soja & $976 \pm 16,7$ & $1034 \pm 15$ & $1005 \pm 12^{A}$ \\
\hline Moyenne & $862 \pm 20^{\mathrm{B} 1}$ & $937 \pm 19^{\mathrm{A}}$ & \\
\hline \multicolumn{4}{|c|}{ Gain de poids vif $(\mathrm{g})$} \\
\hline Aliment coton & $607 \pm 16,4$ & $698 \pm 19,5$ & $653 \pm 14^{\mathrm{B}}$ \\
\hline Aliment soja & $823 \pm 15,6$ & $882 \pm 14,3$ & $853 \pm 11^{A}$ \\
\hline Moyenne & $715 \pm 19^{\mathrm{B}}$ & $790 \pm 18^{A}$ & \\
\hline \multicolumn{4}{|c|}{ Consommation d'aliments ( $\mathrm{g}$ ) } \\
\hline Aliment coton & $1348 \pm 23,5$ & $1502 \pm 26$ & $1425 \pm 21^{B}$ \\
\hline Aliment soja & $1598 \pm 18,4$ & $1691 \pm 19$ & $1644 \pm 15^{\wedge}$ \\
\hline Moyenne & $1473 \pm 23,5^{\mathrm{B}}$ & $1596 \pm 21^{\mathrm{A}}$ & \\
\hline \multicolumn{4}{|c|}{ Indice de consommation } \\
\hline Aliment coton & $2,24 \pm 0,03$ & $2,17 \pm 0,04$ & $2,20 \pm 0,026^{\mathrm{B}}$ \\
\hline Aliment soja & $1,95 \pm 0,04$ & $1,92 \pm 0,02$ & $1,94 \pm 0,021^{A}$ \\
\hline Moyenne & $2,09 \pm 0,030^{\mathrm{A}}$ & $2,05 \pm 0,030^{\wedge}$ & \\
\hline
\end{tabular}

${ }^{1}$ Les moyennes qui figurent sur une même ligne ct celles d'une même colonne et portant la même lettre ne sont pas significativement différentes $(p<0,0001)$.

sur tous les paramètres zootechniques (tableau II). Ainsi, on observe que les animaux nourris avec l'aliment contenant le tourteau de coton présentent un gain de poids vif moyen et un ingéré alimentaire significativement inférieurs, respectivement $-23,4 \%$ et $-13,3 \%$, à ceux des animaux consommant l'aliment à base de tourteau de soja et leur indice de consommation est augmenté de $13,4 \%$.

D’une façon générale, la forme de présentation : granulé vs. farine de l'aliment a un effet significatif $(p<0,0001)$ sur le gain de poids vif $(+10,5 \%)$ et l'ingéré alimentaire de $+8,4 \%$ (tableau $I I$ ). L'indice de consom- mation est également plus faible pour les aliments granulés $(-2,4 \%)$. Pour tous ces paramètres, aucune interaction significative entre la nature des protéines distribuées et la forme de présentation de l'aliment n'est détectée.

\section{DISCUSSION}

Au cours de cette étude, la substitution du tourteau de soja par le tourteau de coton a entraîné des réductions significatives des performances zootechniques des oiseaux alors que les aliments étaient calculés pour 
apporter les mêmes quantités de nutriments bruts et satisfaire les besoins des animaux.

En réalité, les valeurs analytiques se sont révélées supérieures aux valeurs calculées pour couvrir les besoins des animaux et l'aliment soja était par rapport à l'aliment coton, plus concentré en protéines brutes : 21,25 vs. $20,0 \%$, en lysine : 1,17 vs. $1,13 \%$ et en acides aminés soufrés : 1,01 vs. 0,91\% (tableau I).

Une partie des différences de performances observées entre animaux soja et coton pourrait être attribuée à cette composition plus favorable de l'aliment soja. En outre, il est admis que la digestibilité des acides aminés du tourteau de coton est inférieure à celle du tourteau de soja, cette différence peut atteindre $20 \%$ pour la lysine $[2$, $12,13]$. Dans ces conditions, la teneur en lysine disponible ou en autres acides aminés indispensables peut devenir limitante.

Une partie des différences de performances observées entre soja et coton est sans doute aussi à attribuer au gossypol résiduel $[4,15]$, même si le tourteau de coton non granulé utilisé s'est avéré pratiquement peu concentré en ce facteur antinutritionnel $(0,14 \%)$.

Déséquilibre en acides aminés essentiels digestibles et présence de gossypol résiduel $(0,04 \%)$ peuvent expliquer en grande partie les différences de performances observées entre aliments coton et soja granulés. Dans une étude complémentaire, il conviendrait de mieux équilibrer les apports en nutriments digestibles des deux rations présentées en granulé et de rationner les poulets soja au même niveau d'ingestion que les poulets coton afin de quantifier encore plus précisément l'effet spécifique du tourteau de coton.

La granulation a pour effet principal d'accroître la densité de l'aliment [16] et ainsi d'améliorer l'ingestion [3, 7, 16]. Dans nos conditions expérimentales, la granulation a amélioré la densité physique des aliments coton et soja de 25 et $15 \%$ respectivement.
Dans le même temps l'ingéré était accru de 11 et $6 \%$. Les faibles performances observées dans la littérature $[4,6,7]$ lorsque des incorporations élevées (supérieures à $20 \%$ ) de tourteau de coton sont pratiquées sont avant tout liées à une sous-consommation d'aliment induite par une présentation en farine inadaptée.

\section{CONCLUSION}

La granulation des aliments contenant des proportions importantes de tourteau de coton améliore le niveau d'ingestion alimentaire et, par conséquent, les performances zootechniques des poulets de chair. Ce traitement technologique systématiquement utilisé en Europe offre des possibilités d'une meilleure valorisation des tourteaux de coton, source principale de protéines alimentaires pour les volailles en République Centrafricaine, il en est de même de divers produits et sous produits dont la densité nutritionnelle est faible : sons des céréales, racines et tubercules amylacés, pulpes... Améliorant la vitesse de croissance, cette pratique réduirait les durées d'élevages et éventuellement les coûts de production des produits avicoles à condition qu'elle soit économiquement viable. Cependant, quelle que soit la forme de présentation, les performances de croissance obtenues avec les aliments contenant des taux élevés de tourteau de coton n'arrivent pas au niveau de celles obtenues avec l'aliment contenant du tourteau de soja. Plusieurs hypothèses peuvent être évoquées, en particulier la présence de facteurs antinutritionnels dont le gossypol.

La plus plausible est sans doute la moindre disponibilité des acides aminés essentiels, ce point mériterait des études plus approfondies.

\section{REMERCIEMENTS}

Les auteurs remercient Guyomarc'h nutrition animale (Vannes) pour sa contribution au dosage du gossypol. 


\section{RÉFÉRENCES}

[1] Atuahene C.C., Donkoh A., Nkansah-darko P., Effect of cottonseed meal on the performance, carcass characteristics and certain blood parameters of broiler chickens, T. Anim. Prod. Res. 6 (1986) 107-114.

[2] Almquist H.J., Hobart R. Halloran, Bioassay for lysine in cottonseed meal, Feedstuffs, 47 (1975) 39-40.

[3] Calet $C$., The relative value of pellets versus mash and grain in poultry nutrition. World's Poult. Sci. J. 21 (1965) 23-52.

[4] Dongmo T., Effets des régimes alimentaires contenant du tourteau de coton ou du gossypol libre, sur la reproduction des cogs ou des poules (Gallus gallus domesticus), thèse, 1993, $152 \mathrm{p}$.

15] Dongmo T., Pone D.K., Ngoupayou J.D.N., Cottonseed cake in breeder hens diets: effects of supplementation with lysine and methionine, Arch. Geflügelkd. 53 (1989) 231-234.

[6] EL-boushy A.R., Raterink R., Replacement of soybean meal by cottonseed meal and peanut meal or both in low energy diets for broilers. Poult. Sci. 68 (1989) 799-804.

[7] Ergül M., Replacing fish meal with brewers yeast in broiler diets with high levels of cottonseed meal and sunflower seed meal, Land Banforsch. Volkenrode. 38 (1988) 211-219.

[8] Hussar N., Robblee A.R., Effects of pelleting on the utilisation of feed by the growing chickens, Poult. Sci. 41 (1962) 1489-1493.

[9] IEMVT, Manuel d'aviculture en zone tropicale. Ministère des Relations Extérieures, Paris (FRA), 1983, $286 \mathrm{p}$.

[10] Larbier M., Leclercq B., Nutrion et alimentation des volailles, Inra éditions, Paris (FRA), $1992,355 \mathrm{p}$.

[11] McNaughton J.L., Reece F.N., Factors affecting pelleting response. 1. Influence of dietary energy in broiler starter diets, Poult. Sci. 63 (1984) 682-685.

[12] Nwokolo E.N., Brago D.B., Kitts W.D., The availability of amino acids from palm kernel, soybean, cottonseed and rapeseed meal for growing chick. Poult. Sci. 55 (1976) 2300-2304.

[13] Packham R.G., Payne C.G., Cotton seed in broiler diets. Considerations of some aspects of amino acid balance and gossypol level in cottonseed meal and cottonseed meat meal starter diets, Aust. J. Exp. Agric. Anim. Husb. 13 (1973) 656-661.

[14] Plavnik I., Wax E., Sklan D., Hurwwitz S., The response of broiler chickens and turkey poults to steam-pelleted diets supplemented with fat or carbohydrates, Poult. Sci. 76 (1997) 1006-1013.

[15] Pourreza J., Keshavarz., The use of cottonseed meal in broilers diet. Iran Agric. Res. 1 (1982) 181-190.

[16] Rane R.S., Nemade P.P., Advantages of pellet feed and its qualitative importance, Poult. Advis. 29 (1996) 25-27.

[17] Reid B.L., Galaviz-Moreno S., Maiorino P.M. A comparison of glandless and regular cottonseed meals for laying hens, Poult. Sci. 63 (1984) 1803-1809.

[18] Saville D.G., Smith L., Nichols P., Cottonseed meal as a source of protein in diets for laying hens, Aust. J. Exp. Agric. Anim. Husb. 9 (1969) 413-416.

[19] Tacher G., Rivière R., Landry C., L'utilisation du tourteau de coton sans gossypol dans l'alimentation des volailles de chair, in : C.R. Colloque Idessa-CIDT-Trituraf : Le cotonnier sans gossypol : une nouvelle source alimentaire. Abidjan, 26-27 novembre 1985, 97-111.

[20] Yo T., Utilisation directe des graines de coton décortiquées de variétés sans gossypol dans l'alimentation des poulets de chair en Côte-d'Ivoire. Revue Élev. Méd. Vét. Pays trop. 44 (1991) $355-360$. 